\title{
Sobre uma política de ciência e tecnologia para a saúde
}

\author{
On a policy of science and technology for health
}

Reinaldo Guimarães'

DOI: 10.1590/0103-1104201912014

RESUMO Frente ao conjunto de políticas de ciência e tecnologia existentes no Brasil, o texto reivindica um olhar diferenciado sobre a política de pesquisa em saúde. Isso decorre de sua magnitude física, de sua tradição histórica e de sua articulação com uma política pública de saúde na qual a intersetorialidade é valorizada. O texto se divide em três partes, precedidas de uma advertência sobre o impacto da conjuntura atual do País sobre a política geral de ciência e tecnologia. Em primeiro lugar, propõe uma abordagem metodológica para a definição das fronteiras da pesquisa em saúde. Em seguida, reivindica para o campo da saúde coletiva um papel de protagonismo na construção dessa política. Finalmente, apresenta e discute alguns desafios atuais postos para a política.

PALAVRAS-ChAVe Saúde pública. Sistema Único de Saúde. Pesquisa. Política pública. Política de pesquisa em saúde.

ABSTRACT Facing the Brazilian ongoing general science and technology policies, the text claims for a differentiated view on health research policy. This stems from its physical magnitude, its historical tradition, and its articulation with a public health policy in which intersectoriality is valued. The text is presented in three parts, preceded by a warning about the impact of current Brazilian situation on the general policy of science and technology. First, it proposes a methodological approach for the definition of health research boundaries. Then it claims for the field of Collective Health a leading role in the construction of this policy. Finally, it presents and discusses some of the current challenges posed to this politics.

KEYWORDS Public health. Unified Health System. Research. Public policy. Health research policy.

1 Universidade Federal do Rio de Janeiro (UFRJ), Núcleo de Bioética e Ética Aplicada - Rio de Janeiro (RJ), Brasil.

reinaldo.guimaraes47@

gmail.com 


\section{Introdução}

Este texto objetiva trazer novamente à tona um tema que está relativamente fora da pauta, tanto no debate no campo da política de saúde quanto no da ciência e tecnologia. Animada pela realização das I e II Conferências Nacionais de Ciência e Tecnologia em Saúde, respectivamente em 1994 e 2004 - tendo a última incluído mais fortemente na discussão o tema da inovação -, a discussão sobre o lugar e as especificidades da política de pesquisa em saúde no Brasil foi objeto de múltiplas reflexões e práticas desde meados da década de 1980 até o final da primeira década deste século.

A partir do ano 2000, boa parte da bibliografia disponível sobre o tema apresentou e avaliou ações e programas levados a cabo pelo Departamento de Ciência e Tecnologia do Ministério da Saúde (Decit), criado em 2000 1-15. Nos últimos anos, no entanto, o tema perdeu momentum, com a notável exceção do componente do desenvolvimento e inovação industrial em saúde, sustentado pela persistência da Política de Desenvolvimento Produtivo, inaugurada em 2008 no Ministério da Saúde com o apoio do Banco Nacional de Desenvolvimento Econômico e Social (BNDES) e das três versões de política industrial vigentes nos governos dos presidentes Lula da Silva (2003-2010) e Dilma Rousseff (2011-2016). Esse componente, a despeito de sua relevância, estará fora do escopo deste texto por duas razões. A primeira, de ordem prática, é a extensão do tema. A segunda é conceitual e responde à necessidade de alertar para os problemas advindos de um por vezes artificial amálgama entre ciência, tecnologia e inovação, tão em voga nos dias atuais.

O repertório conceitual mais utilizado na análise das condições necessárias ao progresso técnico e o lugar das inovações de base científica e tecnológica nessas condições nasceu nos anos 1980 e foi desenvolvido por vários autores na Europa e nos Estados Unidos na década seguinte. Tem como eixo central o conceito de Sistemas Nacionais de Inovação, que, atualmente, compreende uma extensa família conceitual no campo da economia da tecnologia ${ }^{16}$ que inclui, entre muitos outros, o conceito de sistema setorial de inovação de saúde.

Há, no Brasil, importante literatura sobre este tema ${ }^{17-19}$. Dentre outras aquisições, esse repertório superou a muito tradicional explicação linear na interpretação do progresso técnico, tal como estabelecida nos Estados Unidos da América e daí para o mundo, ao final da II Guerra Mundial. Entretanto, uma leitura talvez menos sofisticada dos conceitos envolvidos na proposta dos Sistemas Nacionais de Inovação provocou um efeito colateral, que foi o de instituir nas mentes da comunidade científica e da sociedade interessada em geral uma tríade compulsória, indissociável e, na minha percepção, reducionista, entre ciência, tecnologia e inovação. Há, por certo, razões para o sucesso dessa leitura, sendo talvez a principal delas o crescimento exponencial das inovações industriais com raízes em investigação científico-tecnológica em todo o mundo. Esse fato produziu um intenso estreitamento espaço-temporal entre o cientista, o tecnólogo e o inovador-empreendedor.

Um dos problemas dessa tríade indissolúvel está em que, em termos globais, a busca do conhecimento científico é majoritariamente fomentada com recursos financeiros e materiais públicos, e isso deveria colocá-lo na categoria de um 'bem público'20, o que nem sempre ocorre quando nos deslocamos da atividade científica para a tecnológica e dessa para a inovação. É muito provável que a disseminação bem-sucedida da tríade venha contribuindo para o enfraquecimento do conceito do conhecimento científico como portador de um caráter eminentemente público.

A mais recente expressão política dessa disseminação - nascida, aliás, no campo da pesquisa em saúde - foi a emergência da noção de 'pesquisa translacional'21. Como comentarei no final deste texto, essa possível diluição do caráter público do conhecimento gerado em pesquisa científica pode estar engendrando possíveis dificuldades no campo ético. 
A esse respeito, e associando conceitualmente a criação científica à invenção, Godin cita Schumpeter quando este último afirma:

innovation is possible without anything we should identify as invention and invention does not necessarily induce innovation. Invention is an act of intellectual creativity and 'is without importance to economic analysis'22(35).

\section{Uma advertência prévia}

Na partida, penso ser indispensável um comentário que, de certo modo, condiciona tudo o que virá depois. Acredito que, a permanecer a conjuntura de destruição do Estado brasileiro e da soberania do País em que vivemos desde 2016 e que, para o campo das políticas sociais, bem como da ciência e tecnologia, tem como mais importante ferramenta normativa a Emenda Constitucional 95 (EC95) - antes denominada Proposta de Emenda Constitucional 241 (PEC 241) -, todas as considerações que farei a seguir não irão muito além de um exercício intelectual inócuo quanto a suas possibilidades de se transformar em ação política.

Os impactos da EC95 sobre as políticas sociais e sobre o financiamento do Sistema Único de Saúde (SUS) têm sido bastante bem estabelecidos, destacando-se o trabalho de Sulpino Vieira e Benevides ${ }^{23}$. No campo específico dos gastos federais em ciência e tecnologia, uma estimativa foi realizada por $\mathrm{mim}^{24}$.

Em termos sintéticos, Sulpino Veira e Benevides mostram que:

A regra vigente de aplicação de recursos em ASPS [ações e serviços públicos de saúde] pelo governo federal estabelece que em 2016 a aplicação mínima deve ser de $13,2 \%$ da $\mathrm{RCL}$ [receita corrente líquida], chegando até $15,0 \%$ da RCL em 2020. Supondo que esta regra estivesse valendo a partir de 2003, sendo aplicado o valor equivalente a 13,2\% da RCL de 2002 para calcular o mínimo daquele ano, a perda entre 2003 e 2015 teria sido de $R \$$ 257 bilhões em comparação com a aplicação realizada no período, cuja regra era dada pela EC2923(9).

Para os gastos federais com ciência e tecnologia, a minha simulação foi distinta. Nesse caso:

Tomando como indicador o aporte de recursos financeiros, os potenciais impactos da PEC 241 sobre a política federal de C\&T podem ser avaliados ao compararmos a curva de crescimento nominal destes recursos entre 2000 e 2013 com a que resulta da curva hipotética quando projetamos a regra da PEC durante o mesmo período [...] Como resultado, temos que os dispêndios financeiros teriam uma variação a menor agregada de $R \$ 79,8$ bilhões em relação ao efetivamente despendido no período $24(2)$.

\section{Sobre a definição de pesquisa em saúde}

Este texto está estruturado em três partes, a saber: (1) uma indagação metodológica sobre a definição da pesquisa em saúde; (2) as relações entre a construção de uma política de pesquisa em saúde e o campo da saúde coletiva; e (3) alguns desafios postos para uma política de pesquisa em saúde hoje em dia.

A reivindicação de um lugar destacado e específico para a pesquisa em saúde no âmbito da política geral de ciência e tecnologia no Brasil decorre de três evidências: a primeira é sua grande dimensão física em termos da massa crítica nela envolvida; a segunda diz respeito à sua mais que centenária existência como prática estabelecida institucionalmente no Brasil, talvez o mais antigo componente setorial de pesquisa; e a terceira vincula-se à sua relevância no âmbito de uma política pública - a política de saúde - na qual o principal instrumento para seu exercício entre nós, o SUS, prevê o cumprimento de intersetorialidade 
como um de seus fundamentos. Estas duas últimas evidências, ambas de caráter histórico, foram discutidas em 'Ciência, tecnologia e inovação: um paradoxo na reforma sanitária', publicado em $2005^{25}$.

Indagar sobre e definir as fronteiras do campo de pesquisa em saúde é um exercício formal, mas não apenas. Em primeiro lugar, porque a escolha de um determinado padrão de inclusão/exclusão/localização de temas científico-tecnológicos na complexa 'árvore' que organiza as áreas do conhecimento no seio da burocracia que governa a atividade de fomento nem de longe é um processo neutro. $\mathrm{E}$, talvez, a maior evidência dessa ausência de neutralidade seja a dificuldade em alterar as 'árvores' em uso, mesmo sabendo que o recorte que as orienta esteja grandemente defasado em relação à dinâmica atual da atividade científico-tecnológica. Essa dificuldade sugere fortemente que, na decisão de conservar o que se sabe ser obsoleto, haja elementos de disputa de poder político difíceis de serem arbitrados. Mas, no caso da pesquisa em saúde, essa questão agrega às dificuldades políticas gerais para introduzir aperfeiçoamentos o fato de dificultar a expressão, no campo da pesquisa, do exercício da intersetorialidade prevista na concepção do SUS.

Inaugurado por trabalhos de Juan Cesar Garcia, de $1986^{\mathbf{2 6}}$, e Julio Frenk et al. ${ }^{27}$, uma reflexão mais sistemática sobre a pesquisa em saúde no Brasil e na América Latina pertence aos anos 1990 28-37. Nesse amplo conjunto de trabalhos, quase sempre que uma delimitação do campo da pesquisa em saúde foi necessária, o recorte baseou-se numa concepção de identificar essa atividade como o somatório da pesquisa em biociências, pesquisa clínica, epidemiologia e saúde pública.

O modo habitual de delimitar o campo da pesquisa em saúde entre nós e nas bases de dados internacionais (Web of Science, Scopus, Thomson Reuters etc.) realiza-se a partir do somatório das publicações nas grandes áreas das ciências biológicas e das ciências da saúde, e isso não reflete de modo correto o que ela é. A inadequação da delimitação atual pode ser verificada quando, em números extraídos do Diretório dos Grupos de Pesquisa do Conselho Nacional de Desenvolvimento Científico e Tecnológico (CNPq) em sua base corrente de dados, se observa o número de linhas de pesquisa vinculadas à saúde humana em grupos de pesquisa segundo as grandes áreas do conhecimento que orientam sua atividade predominante. Embora, como seria de se esperar, a maioria das linhas pertençam a grupos das ciências biológicas e das ciências da saúde, restam mais de seis mil linhas oriundas de todas as demais grandes áreas do conhecimento constantes na árvore (figura 1). 
Figura 1. Linhas vinculadas ao setor 'saúde humana' segundo a grande área predominante do grupo de que fazem parte
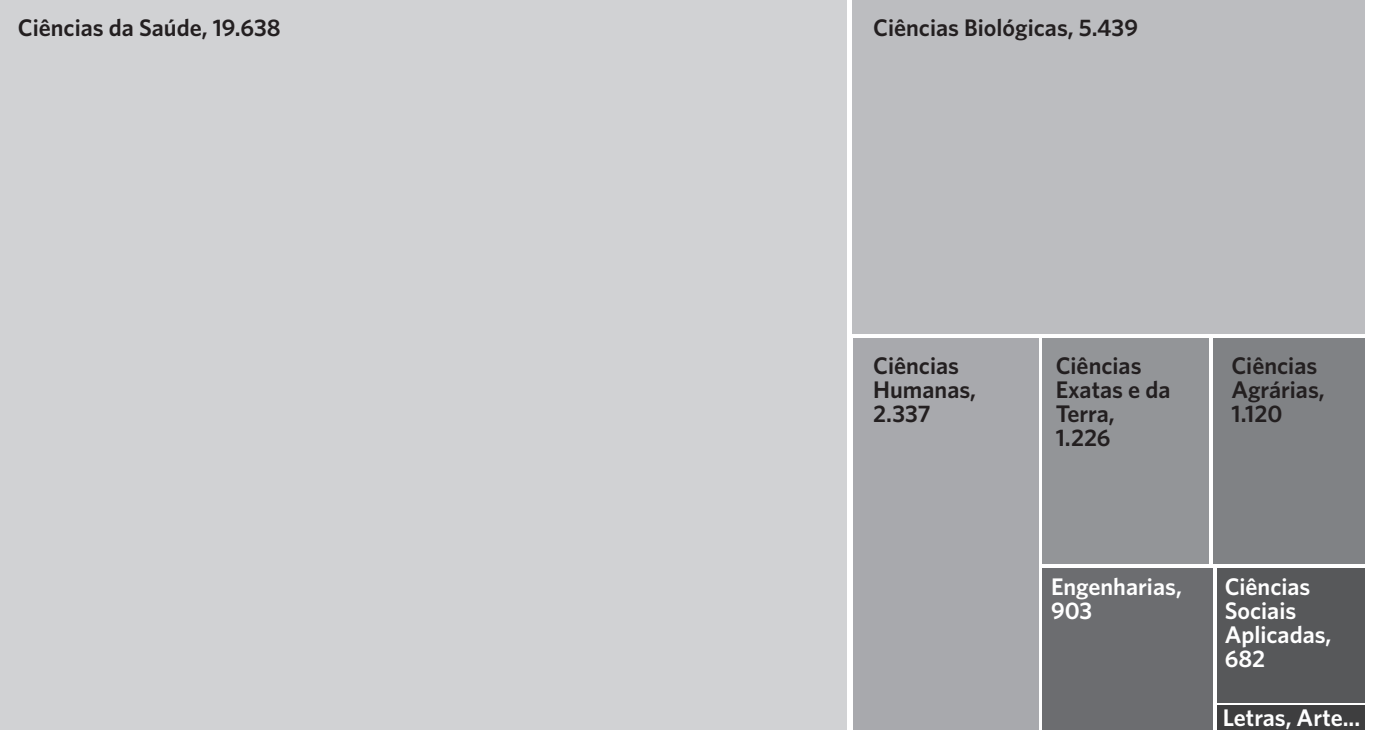

Uma delimitação adequada da pesquisa em saúde sugere entendê-la como um 'setor de atividade ou de aplicação', diferentemente de outros campos de saber científico-tecnológico, cujas bases epistemológicas são estabelecidas por parâmetros teórico-conceituais (física, química, biologia etc.) ou disciplinares (engenharias, medicina, direito etc.). Numa perspectiva, que poderíamos estabelecer como teleológica, a pesquisa em saúde define-se como a pesquisa destinada a atuar positivamente sobre o estado de saúde dos indivíduos ou coletivos humanos ${ }^{38}$. De modo sintético, a pesquisa em saúde poderia ser denominada como a 'pesquisa para a saúde'.

Reitero que essa questão está longe de ser apenas contábil e formal. No plano conceitual, a delimitação tradicional encerra uma determinada visão da pesquisa em saúde que colide, como veremos a seguir, com a perspectiva epistemológica da saúde coletiva. Do ponto de vista da construção de uma política para a pesquisa em saúde, ela reduz sua amplitude. Do ponto de vista do exercício dessa política, ela prejudica a porosidade entre saberes sanitários, biológicos, sociais, tecnológicos, clínicos, epidemiológicos, psicológicos etc., grandemente necessária ao bom êxito dessa política.

\section{A política de pesquisa em saúde e o campo da saúde coletiva}

O setor público de saúde no Brasil possui uma importante tradição institucional histórica de pesquisa científica e tecnológica em unidades próprias, federais e estaduais. Aquela talvez mais remota data do ano de 1900, com a fundação do Instituto Oswaldo Cruz, hoje pertencente à Fundação Oswaldo Cruz (Fiocruz), à qual se seguiu a fundação do Instituto Butantan, em 1901, e de outros institutos similares, principalmente no estado de São Paulo. Os dois institutos pioneiros tiveram como missões originais o desenvolvimento e fabricação de soros para as campanhas de saúde pública da época. Neles, a atividade de pesquisa científica 
acompanhou essa vertente tecnológica primordial, mas sempre sem deixar de valorizar a pesquisa científica original.

Entretanto, no Brasil, ao longo de quase todo o século XX, o estímulo à manutenção de vínculos entre a pesquisa e as ações de saúde pública foi quase sempre influenciado pela presença ubíqua da Fundação Rockefeller na saúde pública, nas cidades e sertões brasilei$\operatorname{ros}^{39}$. E seu padrão de cultura institucional, persistente em instituições brasileiras mesmo após a saída daquela fundação do País, diferentemente da tradição dos pioneiros do início do século $\mathrm{XX}$, valorizava quase exclusivamente a pesquisa operacional de avaliação de programas e ações de saúde, deixando de lado a pesquisa original tão presente no modelo de Oswaldo Cruz, Carlos Chagas, Adolpho Lutz e outros. O principal herdeiro da tradição institucional da Fundação Rockefeller no Brasil e grande cultivador dessa orientação para a atividade de pesquisa em saúde foi o Serviço Especial de Saúde Pública (Sesp), criado em 1942 nos marcos de um tratado bilateral BrasilEstados Unidos ${ }^{40}$.

Como parte desse panorama, vale também acrescentar que o divórcio entre a pesquisa científica original e os serviços de saúde teve como um combustível adicional a emergência avassaladora, em todo o mundo, da explicação linear do progresso técnico a partir do final da II Guerra Mundial. Esse modelo explicativo elegeu a pesquisa básica como variável autônoma e crítica para o avanço do conhecimento e para o progresso técnico ${ }^{\mathbf{4 1}}$, bem como também elegeu o modelo norte-americano de universidade de pesquisa como a instituição-padrão para o exercício dessa pesquisa 'desinteressada' de aplicações práticas. Em linhas muito gerais, essa dupla determinação estabeleceu o retrato das relações entre as políticas de saúde e a pesquisa original em saúde nas últimas seis ou sete décadas: caminhos autônomos e institucionalmente separados.

A missão da saúde coletiva, de pensar e propor uma política de Ciência \& Tecnologia (C\&T) em saúde, possui uma história 'grosso modo' contemporânea à história do SUS. Poderíamos considerar como marco inaugural de uma relação formalizada entre a saúde coletiva e a pesquisa em saúde, a I Conferência de Ciência e Tecnologia em Saúde, convocada pelo Conselho Nacional de Saúde e realizada em outubro de 1994 sob os auspícios dos Ministérios da Saúde, da Ciência e Tecnologia e da Educação. As resoluções da conferência foram vítimas das vicissitudes da troca de governo ocorrida dois meses depois e que fizeram com que várias de suas decisões e recomendações fossem ignoradas ou postergadas pelo novo governo. Entretanto, duas delas, centrais para o tema da política de ciência e tecnologia em saúde, sobreviveram.

Uma delas, de caráter conceitual, estabelecia que 'a política de ciência e tecnologia em saúde é um componente da política nacional de saúde', e essa assertiva está nas bases da reflexão que aqui está sendo feita. A outra, de caráter institucional, propunha a criação de uma Secretaria de Ciência e Tecnologia no Ministério da Saúde.

Esta última só foi se concretizar em 2003, quase dez anos depois. Em 2004, houve a II Conferência, também em Brasília, e que agregou duas contribuições importantes ao tema. A primeira foi a de introduzir a questão da inovação tecnológica e da necessidade de uma política para o componente industrial da saúde no ambiente do gestor federal do SUS, praticamente ausente desde o declínio do programa da Central de Medicamentos. A segunda, a de formular uma política explícita de pesquisa em saúde, baseada nos princípios gerais que animaram a I Conferência, bem como aprovar uma agenda de prioridades de pesquisa, cuja proposta foi elaborada em um grande encontro de pesquisadores, gestores, profissionais de saúde e usuários dos serviços que contou com a participação de cerca de 500 pessoas no final de $2003^{42}$. Infelizmente, em 2014 não houve condições de se realizar a III Conferência, e ela foi substituída por um Seminário realizado na Fiocruz, no Rio de Janeiro. 
É pertinente indagar por que caberia ao campo da saúde coletiva formular uma política de pesquisa em saúde para o País, e não apenas para os espaços tradicionais da saúde pública, da medicina preventiva e da medicina social. Esse papel se justifica e se estrutura na própria definição da saúde coletiva e naquilo que a diferencia daqueles espaços tradicionais. Os idealizadores das definições básicas da saúde coletiva tiveram, desde sempre, uma visão ampla de seu escopo, e essa visão foi sistematizada na sua definição como um campo de conhecimento e um âmbito de práticas.

Se a política de pesquisa em saúde deve ser interpretada como um componente da política de saúde e se a política de saúde é um dos objetos privilegiados da reflexão e da prática da saúde coletiva, então cabe a esta incorporar em sua missão a tarefa essencial de pensar e propor sobre a política de pesquisa como um todo, e não apenas nos seus componentes tradicionalmente vinculados à saúde pública. Em termos de organização temática, essa visão ampliada deve compreender como áreas de investigação as transições saúde-doença (promoção, prevenção, cura, reabilitação), aí incluídos os mecanismos básicos, individuais e coletivos que as determinam; os sistemas e as políticas de saúde; a intersetorialidade na saúde e as relações entre saúde e sociedade.

Deriva daí que uma política de pesquisa para a saúde deve abraçar todos os seus componentes, a saber: biomédico, clínico, epidemiológico, bem como as originárias no campo das ciências sociais, aqui incluídos o planejamento e a gestão no campo da saúde, sem quaisquer reducionismos.

\section{Desafios para uma política de pesquisa em saúde}

É consenso que o amadurecimento do sistema setorial de inovação em saúde no Brasil depende, entre outros aspectos, de uma presença bem maior de estímulos da demanda por produtos e serviços em relação à oferta de conhecimento, e isso obriga a pensar em dois movimentos que, idealmente, deveriam ser simultâneos e complementares. No plano da criação científica e tecnológica, isso remete à necessidade de uma aproximação muito mais intensa por parte dos cientistas dos órgãos responsáveis pela demanda de cerca de um terço do mercado de medicamentos, de $90 \%$ do mercado de vacinas, de $50 \%$ do mercado de equipamentos de saúde, de $100 \%$ dos serviços prestados a todos os brasileiros por mandato constitucional e a $75 \%$ deles que dependem exclusivamente do SUS.

Simetricamente, os formuladores e gestores da política pública de saúde deveriam compreender a importância de uma aproximação muito mais intensa com a comunidade científica e tecnológica no setor de saúde. Não como um elemento relativamente acessório e eventual, mas como um componente constitutivo essencial da política de saúde, tal como preconizou a conferência de 1994. Mais ainda, considerando o essencial papel da demanda na constituição de um sistema setorial de inovação em saúde, no qual o conhecimento científico tem grande participação nos dias de hoje, é aos formuladores e gestores da política que cabe induzir o movimento de aproximação do mundo da pesquisa em saúde.

Mas não se trata de qualquer aproximação. Como foi esboçado mais acima na apresentação das relações históricas entre pesquisa e serviços de saúde no Brasil, os vínculos predominantes entre pesquisa em saúde e política de saúde foram governados por uma visão excessivamente utilitária das aquisições científicas e tecnológicas a essa política. A manutenção desse padrão de relação implicaria um reducionismo que seria o de circunscrever os esforços da criação científica e tecnológica às necessidades imediatas dos gestores do SUS. Se a pauta da pesquisa que a saúde coletiva propõe é uma pauta ampliada, é essa pauta que os gestores do SUS deveriam abraçar e que deveria governar a aproximação desses dois mundos.

Seja pela óptica da atividade de pesquisa, seja pela da pós-graduação, o setor de saúde 
humana destaca-se no cenário do esforço de pesquisa científica e tecnológica no Brasil. A saúde humana detém o maior número de programas de pós-graduação, bem como a maior massa crítica, medida por pesquisadores envolvidos com linhas de pesquisa nesse setor. De acordo com os dados disponíveis no CNPq, a saúde humana é o objeto de trabalho de $34,1 \%$ dos pesquisadores com titulação doutoral no País (50.286 em 147.647) ${ }^{43}$ e, segundo a Coordenação de Aperfeiçoamento de Pessoal de Nível Superior (Capes)/Geocapes, 21,8\% dos programas de pós-graduação stricto sensu (912 em 4.177 programas). Esse dado da Capes resulta da soma dos programas das áreas de medicina, ciências biológicas e psicologia. O Geocapes não permite a identificação dos programas envolvidos com saúde humana em programas das grandes áreas de ciências exatas e da terra, agrárias e ciências humanas e sociais. Portanto, a participação do setor de saúde humana apresentado no texto implica algum grau de subestimação. Essas dimensões autorizam pensar em um olhar diferenciado para ela.

Entendo que esse olhar diferenciado deveria se refletir não apenas na dimensão dos aportes financeiros, mas também em outros aspectos da atividade de apoio à pesquisa. Nos países líderes globais na pesquisa em saúde, como Estados Unidos, Reino Unido, França, Canadá e Espanha, entre outros, esse olhar diferenciado se expressa, ao lado do volume de recursos financeiros aportados, também na organização institucional do fomento, que se apoia em entidades especializadas no fomento à pesquisa em saúde. A localização dessas organizações na estrutura institucional governamental é variada, sendo elas vinculadas aos ministérios de Desenvolvimento e Indústria, de Ciência e Tecnologia e da Saúde, em múltiplas formas de divisão de tarefas. Acredito que para o desenvolvimento de uma política pública de pesquisa em saúde no Brasil, seria de grande valia a criação de uma estrutura desse tipo.

As dificuldades políticas e burocráticas envolvidas na aplicação desse modelo no Brasil são muito grandes, o que sugere talvez um caminho de avanços incrementais. Por exemplo, o estabelecimento de acordos duradouros entre as agências de fomento e outros componentes organizacionais já existentes nos ministérios da Saúde, da Ciência, Tecnologia e Inovação e da Educação, além dos Conselhos das fundações estaduais de apoio à pesquisa, de cogestão - inclusive cofinanciamento - de programas e projetos de pesquisa.

Já ocorreram neste século experiências estimulantes nesse sentido. Por exemplo, a participação do Ministério da Saúde na seleção e financiamento na primeira chamada dos Institutos Nacionais de Ciência e Tecnologia (INCTs), o Programa Pesquisa para o SUS (PPSUS), gerenciado pelo Departamento de Ciência e Tecnologia do Ministério da Saúde, $\mathrm{CNPq}$, fundações de apoio à pesquisa e secretarias estaduais de Ciência e Tecnologia, além do cofinanciamento de projetos relevantes realizados pelo Ministério da Saúde e a Financiadora de Inovação e Pesquisa - Finep (Estudo Longitudinal de Saúde do Adulto, Rede Nacional de Terapia Celular etc.). A fragilidade dessas iniciativas está na inexistência de instâncias mais formalizadas e permanentes de coordenação, sempre dependentes de vontades de dirigentes de ocasião. Penso, no entanto, que o fortalecimento dessas interações, de modo integrado e permanente - por exemplo, com a criação de um conselho dedicado ao tema composto por todas as agências - teria grande impacto na política de pesquisa em saúde.

Outra característica dessas organizações de fomento à pesquisa em saúde pelo mundo afora é, além de realizarem fomento a grupos externos, possuírem institutos e outros tipos de unidades como componentes de sua estrutura. Menciono esse aspecto para sublinhar o papel da Fiocruz numa trajetória como essa que estou sugerindo. Por sua relevância no ambiente de pesquisa em saúde no Brasil e no exterior, e sua vinculação ao Ministério da Saúde, penso que a Fiocruz poderia ter papel central no aperfeiçoamento e implantação dessas propostas. A própria conformação institucional atual da Fiocruz, com sua ampla 
diversidade de unidades praticantes de, talvez, todas as modalidades de pesquisa em saúde, reforça a proposta de ela vir a desempenhar esse papel.

Penso ainda que aprofundar o conhecimento sobre o perfil da pesquisa em saúde no País é um requisito importante na construção de uma política para ela, e acredito que para isso a Fiocruz também está em posição privilegiada. Por exemplo, atualmente, nosso conhecimento sobre os fluxos de recursos financeiros para a pesquisa em saúde é grandemente insatisfatório. Até onde vai o meu conhecimento, houve uma interessante iniciativa em 2006, liderada por pesquisadores do Instituto de Medicina Social da Universidade do Estado do Rio de Janeiro (Uerj), de construir um modelo para acompanhar esse tema, que foi, infelizmente, descontinuada ${ }^{44}$. Sobre esse e outros aspectos, sugiro com entusiasmo que a Fiocruz abrace essa tarefa.

A partir do modelo analítico proposto neste texto, arrisco, com a cautela necessária ao exercício de prospecções em ciência, sugerir alguns caminhos que me parecem portadores de futuro numa agenda de pesquisa para a saúde no Brasil.

No campo da pesquisa biomédica, um dos grandes desafios tem sido a dificuldade de decifrar a complexidade de enfermidades que cada vez mais se colocam como responsáveis por grande parte da carga de doenças em todo o mundo. E uma das propostas apresentadas para superar essa dificuldade é a da substituição dos modelos animais por modelos humanos de experimentação em pesquisa pré-clínica ${ }^{45}$. $\mathrm{O}$ desenvolvimento desse tema, muito provavelmente, crescerá de importância nos próximos anos e será indispensável o balanceamento dos impactos éticos envolvidos.

Em outra vertente, os caminhos já abertos pelas tecnologias de edição de genes (CRISPRcas $^{9}$ ), bem como o avanço no conhecimento dos mecanismos de diferenciação celular, oferecem larga estrada para a pesquisa biomédica. Esses desafios são globais e nossa inserção nessa dinâmica científica implicará adequada seleção de alvos articulados com nossa nosologia e com nossas perspectivas de liderança e solidariedade com o mundo menos desenvolvido que nós. A recente participação da biomedicina brasileira no surto de zika é um bom exemplo dessa adequação.

No terreno da epidemiologia e da pesquisa clínica, um grande desafio nos tempos à frente parece ser a utilização, como fonte de dados para pesquisa, de grandes bases cuja construção se deu fora do mundo da pesquisa (Big Data) ${ }^{\mathbf{4 6}, 47}$. O desafio está não apenas no aperfeiçoamento de tecnologias capazes de garantir a qualidade das informações dessas bases de dados aos requisitos de investigação científica, como também na construção de uma regulamentação ético-legal capaz de beneficiar a ciência sem colocar em risco os direitos de privacidade dos cidadãos. Em outra clave, entendo que a epidemiologia brasileira vem mudando de patamar com a disseminação de grandes estudos de coorte, desde a pioneira família de coortes de Pelotas até uma das mais recentes e notáveis, que é o multicêntrico 'Estudo Longitudinal de Saúde do Adulto'. Aqui, complementarmente aos desafios intelectuais e logísticos envolvidos, será necessária uma preocupação especial com a sustentabilidade financeira de cada coorte.

Uma das principais marcas das ciências sociais neste século tem sido a avassaladora incorporação, em seu repertório analítico, de dimensões sociais outrora menos valorizadas. Essas dimensões, algumas delas identitárias, como gênero e etnia, cada vez mais se articulam com outras mais assentadas, como por exemplo, classe social, trabalho, educação e religião, para uma melhor compreensão do fenômeno social ${ }^{\mathbf{4 8}}$. Para os estudiosos das relações entre saúde e sociedade, em particular os pesquisadores no terreno da determinação social da saúde e da doença, essas aquisições são essenciais.

Pari passu com a crise do Estado de BemEstar Social, os sistemas públicos universais de saúde estão sob ataque há bem 30 anos ou mais. Nosso SUS está sob ataque desde a sua 
concepção, com a Constituição de 1988. Por outro lado, entendo que nele existem espaços de aperfeiçoamento, avanços que não foram logrados mesmo nos melhores tempos de sua gestão. Não tenho a ilusão de que a pesquisa seja capaz de, isoladamente, reverter essa tendência. Mas acho que aos pesquisadores no campo dos sistemas e políticas de saúde cabe hoje e caberá amanhã fornecer aos atores políticos as evidências necessárias tanto para defender o SUS dos ataques de seus adversários, quanto para colaborar com seus defensores a superar suas deficiências.

Nos dias de hoje e nos que virão, uma política de pesquisa para a saúde, seja no Brasil ou em qualquer parte, deve conter um capítulo específico para os desafios éticos cada vez mais colocados à frente da atividade da investigação científica e tecnológica. Há evidências empíricas de que nos últimos anos são crescentes as agressões à integridade da pesquisa científica, quando medidas pela frequência de retratações de artigos publicados ${ }^{49,50}$ e essas agressões se concentram na pesquisa no campo da saúde, especialmente biomédico. As motivações são variadas, muitas vezes decorrendo da busca de prestígio e das consequentes recompensas financeiras na forma de novos grants. Mas não se deve excluir como determinante a intersecção dos caminhos da pesquisa com a apropriação de suas descobertas pela indústria e pelos serviços.

No quadro da emergência da chamada 'pesquisa translacional', é essencial desenvolver mecanismos capazes de mitigar a emergência do que se poderia chamar de um imperativo translacional'51, mecanismos que previnam a ocorrência de problemas colocados por essa 'translação'. Não apenas os problemas embutidos no objeto e na metodologia da pesquisa, mas também no olhar de seus autores com relação ao destino (comercial/industrial) de seus resultados.

Como já observado no início deste texto, a transformação acrítica de pesquisadores em empreendedores-inovadores, ao encurtar ou mesmo eliminar a distância entre as regras do fazer científico das do fazer industrial e comercial, pode levar a graves problemas, tanto na integridade científica, quanto na legitimidade ética de certas utilizações de seus resultados.

\section{Colaboradores}

Guimarães R (0000-0002-0138-9594)*é responsável pela elaboração do manuscrito. 


\section{Referências}

1. Tess BH. Ciência, tecnologia e inovação em saúde: desafios. Rev. de Direito Sanitário. 2004; 5(2):9-21.

2. Guimarães R. Bases para uma política nacional de ciência tecnologia e inovação em saúde. Ciênc. Saúde Colet. 2004; 9:375-387.

3. Guimarães R. Setting and implementing a health research priority agenda in Brazil. Global Forum Update on Research for Health. 2004; 2:096-098.

4. Guimarães R. O Ministério da Saúde e a pesquisa no Brasil. Rev. Soc. Bras. hipertensão. 2005; 8(4):126-130.

5. Elias FTS, Souza LPF. Indicadores para monitoramento de pesquisa em saúde no Brasil. Ci. Inf. 2006; 35(3):218-226.

6. Guimarães R. Pesquisa em saúde no Brasil: contexto e desafios. Rev. Saúde Pública. 2006; 40(esp):3-10.

7. Guimarães R, Carvalheiro JR, Degrave W. Global forum for health research, monitoring financial flows for health research 2005. In: Burk MA, Francisco A, organizadores. Global Forum for Health Resarch Report. 2006. p. 49-56.

8. Goldbaum M, Serruya SJ. O Ministério da Saúde e a política de ciência, tecnologia e inovação em saúde. Cad. Saúde Pública. 2006; 22(3):470-471.

9. Guimarães R, Santos LMP, Angulo-Tuesta A, et al. Defining and implementing a national policy for technology and innovation in health: lessons from Brazilian experience. Cad. Saúde Pública. 2006; 22:(9):705-731.

10. Goldbaum M, Serruya SJ. O Ministério da Saúde na política de ciência, tecnologia e inovação em saúde. Rev. USP. 2007; 73:40-47.

11. Santos LMP, Souza LEPF, Serruya SJ, et al. O papel da pesquisa na consolidação do Sistema Único de Saúde (SUS). Cad. Saúde Pública. 2010; 26(9):1666-1667.

12. Guimarães R. Desafios da pós-graduação em saúde humana no Brasil. Rev. Saúde Pública. 2011; 45:1-13.

13. Santos LMP, Moura EC, Barata RCB, et al. Fulfillment of the Brazilian agenda of priorities in health research. Health res. policy syst. 2011; 9:35.

14. Paula AP, Giozza SP, Pereira MZ, et al. Clinical investigations for SUS, the Brazilian public health system. São Paulo. Med J. 2012; 130(3):179-186.

15. Moura EC, et al. Research on chronic noncommunicable diseases in Brazil: meeting the challenges of epidemiologic transition. Rev. Panam. Salud Pública. 2012; 31: 240-245.

16. Freeman C. Continental, national and sub-national innovation systems: complementarity and economic growth. Res. Policy [internet]. 2011 [acesso em 2018 maio 16]; 31(2002): 191-211. Disponível em: http:// debis.deu.edu.tr/userweb//sedef.akgungor/dosyalar/freeman.pdf.

17. Cassiolato JE, Albuquerque EM. As especificidades do sistema de inovação do setor saúde. Rev. Econ. Política. [internet] 2002 out [acesso em 2018 maio 26]; 22(4):134-151. Disponível em: https://www.researchgate.net/profile/Jose_Cassiolato/publication/240631812_As_Especificidades_do_Sistema_de_Inovacao_do_Setor_Saudel/ links/54348bc20cf294006f735c3d.pdf.

18. Gadelha CAG, Quental C, Fialho BC. Saúde e inovação: uma abordagem sistêmica das indústrias da saúde. Cad. Saúde Pública. [internet] 2003 [acesso em 2018 maio 26]; 19(1):47-59. Disponível em: https:// www.scielosp.org/pdf/csp/2003.v19nl/47-59/pt.

19. Motta EA, Souza SGA, Baessa AR. Pesquisa e inovação em saúde: uma discussão a partir da literatura sobre economia da tecnologia. Ciênc. Saúde Colet. [internet] 2004 [acesso em 2018 maio 26]; 9(2):277294. Disponível em: http://www.scielo.br/pdf/csc/ v9n2/20385.pdf.

20. Dalrymple D. Scientific knowledge as a global pu- 
blic good: contributions to innovation and the economy. In: The Role of Scientific and Technical Data and Information in the Public Domain. [internet] 2003 [acesso em 2018 maio 5]. Disponível em: https://www.nap.edu/read/10785/chapter/6.

21. Guimarães R. Pesquisa translacional: uma interpretação. Ciênc. Saúde Colet. [internet] 2013 [acesso em 2018 maio 26]; 18(6):1731-1744. Disponível em: http:// www.scielo.br/pdf/csc/v18n6/24.pdf .

22. Godin B. Innovation: the history of a category. Project on the intellectual history of innovation. Working Paper [internet] 2008 [acesso em 2018 maio 26]. Disponível em: http://www.csiic.ca/PDF/IntellectualNol. pdf.

23. Vieira FS, Benevides RPS. Os impactos do novo regime fiscal para o financiamento do Sistema Único de Saúde e para a efetivação do IPEA. In: Vieira FS, Benevides RPS, organizadores. Direito à Saúde no Brasil. Nota Técnica. [internet] 2016 [acesso em 2018 maio 8]. Disponível em: http://www.ipea.gov.br/portal/index.php?option=com $\_$content\&id= 28589 .

24. Guimarães R. A proposta de Emenda Constitucional 241/2016 e o Sistema Único de Saúde: impactos na pesquisa e na indústria. Cad. Saúde Pública. [internet] 2016 [acesso em 2018 maio 5]; 32(12). Disponível em: http://www.scielo.br/pdf/csp/v32n12/16784464-csp-32-12-e00182816.pdf.

25. Guimarães R. Ciência, tecnologia e inovação: um paradoxo na reforma sanitária. In: Lima NT, Gerschman S, Edler FC, et al., organizadores. Saúde e democracia: histórias e perspectivas do SUS. Rio de Janeiro: Fiocruz; 2005. p. 235-256.

26. Garcia JC. La investigación en salud en 11 países de América Latina. Washington, DC: OPAS; 1986.

27. Frenk J, Bobadilla JL, Sepúlveda J, et al. Un modelo conceptual para la investigación en salud pública. Bol Oficina Sanit Panam. 1986; 101:477-492.

28. Pellegrini A. Investigación en salud en América Latina. Washington, DC: OPAS/OMS; 1991.
29. Levcovitz E, Batista TWF, Uchôa SAC, et al. Saberes e políticas: a contribuição do campo da saúde coletiva na organização da política de saúde brasileira. Rio de Janeiro: IMS/UERJ; 2000. [Série Estudos em Saúde Coletiva].

30. Viacava F, Pacheco MF, Porto SM, et al. Perfil e tendências da produção científica em saúde no Brasil. Rio de Janeiro: Centro de Informações para a Saúde/ Fundação Oswaldo Cruz; 1991. v. 2

31. Organização Pan-Americana de Saúde. La investigación em salud en América Latina. Washington DC: OPAS; 1992.

32. Guimarães R, Tavares R, organizadores. Saúde e sociedade no Brasil nos anos 80. Rio de Janeiro: Relume-Dumará; 1994.

33. Guimarães R, Viana CMM. Ciência e tecnologia em saúde: tendências mundiais, diagnóstico global e estado da arte no Brasil. In: Conferência Nacional de Ciência e Tecnologia em Saúde. Anais da $1^{\text {a }}$ Conferência de Ciência e Tecnologia em Saúde; 1994. Brasília, DF: Coordenação Geral de Desenvolvimento Científico e Tecnológico; 1994. p. 115-235.

34. Pellegrini A, Goldbaum M, Silvi J. Producción de artículos científicos sobre salud en seis países de América Latina, 1973 a 1992. Rev Panam Salud Pública. 1997; 1:23-34.

35. Sánchez D, Bazzani R, Gómez S, organizadores. Prioridades en la investigación de la salud colectiva en América Latina. Montevideo: GEOPS; 1998.

36. Pereira JCR, Escuder MML. The scenario of Brazilian health sciences in the period of 1981 to 1995. Scientometrics. 1999; 45:95-105.

37. Coimbra CEA. Produção científica em saúde pública e as bases bibliográficas internacionais. Cad. Saúde Pública. 1999; 15:883-888.

38. Guimarães R. Ciência, tecnologia e pesquisa em saúde. In Giovanella L, organizadora. Políticas e Sistemas de Saúde no Brasil. Rio de Janeiro: Fiocruz; 2008. p. 287. 
39. Santos LAC. O pensamento sanitarista na Primeira República: uma ideologia de construção da nacionalidade. Dados. Rev. de Ciências Sociais. [internet] 1985 [acesso em 2018 maio 16]; 28(2):193-210. Disponível em: http://www.bvshistoria.coc.fiocruz.br/lildbi/docsonline/antologias/eh-594.pdf .

40. Vilarino MTB. Os antecedentes do Serviço Especial de Saúde Pública (SESP) no Brasil, o espaço de experiência, a rede de interdependências e o horizonte de expectativas. In: XX Encontro Regional de História; 2016 [acesso em 2018 maio 16] Disponível em: http://encontro2016.mg.anpuh.org/resources/ anais/44/1472058921_ARQUIVO_Osantecedentes doServicoEspecialdeSaudePublica(SESP)noBrasil Texto_Anpuh2016.pdf.

41. Bush V. A Report to the President by Vannevar Bush, Director of the Office of Scientific Research and Development. [internet]. Washington, DC: United States Government Printing Office; 1945 [acesso em 2018 fev 1]. Disponível em: https://www.nsf.gov/od/lpa/ nsf50/vbush1945.htm.

42. Brasil. Ministério da Saúde. Agenda Nacional de Prioridades de Pesquisa em Saúde. 2. ed. [internet] 2015 [acesso em 2018 maio 15]. Disponível em: http://bvsms.saude.gov.br/bvs/publicacoes/agenda_nacional_ prioridades_2ed_4imp.pdf.

43. Centro Nacional de Desenvolvimento Cientifico e Tecnológico. Diretório dos Grupos de Pesquisa no Brasil. Base corrente [internet]. 2018 [acesso em 2018 maio 17]. Disponível em: http://dgp.cnpq.br/dgp/faces/consulta/consulta_parametrizada.jsf.

44. Vianna CMM, Caetano R, Ortega JA, et al. Flows of financial resources for health research and development in Brazil, 2000-2002. Bulletin of the World Health Organization [internet]. 2007 [acesso em 2018 maio 16]; 85(2):124-130. Disponível em: http://apps.who.int/ iris/bitstream/handle/10665/269896/PMC2636283. pdf? sequence $=1 \&$ is Allowed $=y$.

45. Langley GR, Adcock IM, Busquet F, et al. Towards a 21st-century roadmap for biomedical research and drug discovery: consensus report and recommen- dations. Drug Discov Today. [internet] 2017 [acesso em 2018 maio 21]; 22(2):327-339. Disponível em: https://research.aston.ac.uk/portal/files/20945365/ Roadmap_for_biomedical_research_and_drug_discovery.pdf.

46. Khoury MJ, Ioannidis JPA. Big data meets public health: Human well-being could benefit from large-scale data if large-scale noise is minimized. Science. [internet] 2014 [acesso em 2018 maio 21]; 346(6213):10541055. Disponível em: https://www.ncbi.nlm.nih.gov/ pmc/articles/PMC4684636/.

47. Mooney SJ, Westreich DJ, El-Sayed AM. Epidemiology in the era of big data. Epidemiology. [internet] 2015 [acesso em 2018 maio 21]; 26(3):390-394. Disponível em: https://www.ncbi.nlm.nih.gov/pmc/articles/PMC4385465/.

48. Minayo MCS, Coimbra Junior CEA, organizadores. Críticas e atuantes: ciências sociais e humanas em saúde na América Latina [internet]. Rio de Janeiro: Fiocruz; 2005. [acesso em 2018 maio 21]. Disponível em: https://static.scielo.org/scielobooks/w5p4j/pdf/ minayo-9788575413920.pdf.

49. Cokol M, Rodriguez-Esteban R, Rzhetsky A. Retraction rates are on the rise. Embo Reports [internet]. 2008 [acesso em 2018 maio 22]; 9(1):1. Disponível em: http://embor.embopress.org/content/ embor/9/1/2.1.full.pdf.

50. Steen RG. Retractions in the scientific literature: do authors deliberately commit research fraud? J Med Ethics [internet] 2010 [acesso em 2018 maio 22]; 1-5. Disponível em: http://citeseerx.ist.psu.edu/viewdoc/ download?doi=10.1.1.459.2815\&rep=repl\&type=pdf.

51. Maienschein J, Sunderland M, Ankeny RA, et al. The ethos and ethics of translational research. Am J Bioeth. 2008; 8(3):43-51.

Recebido em 22/08/2018

Aprovado em 21/01/2019

Conflito de interesses: inexistente

Suporte financeiro: não houve 